\title{
AGE EFFECTS IN GIANT EXTRAGALACTIC HII REGIONS
}

\author{
J. Masegosa and M. Moles \\ Instituto de Astrofísica de Andalucía, Granada. Spain
}

\begin{abstract}
Spatial inhomogeneities for a sample of Giant Extragalactic HII Regions (GEHR) have been analyzed. The results indicate that, whitin the observational errors, the $\mathrm{O} / \mathrm{H}$ and $\mathrm{He} / \mathrm{H}$ abundance ratios are constant for a given GEHR. The $\mathrm{Ne} / \mathrm{O}$ ratio on the other hand shows a trend with the evolution of the ionizing stellar clusters.

Spectral changes between different zones cannot be explained in terms of effective temperature variations. We found out that they could be accounted for as differences in the ionization parameter. This parameter, governing the spatial peculiarities in GEHR, is probably related to the age of the ionizing clusters, a fact not yet taken into account in photoionization models.
\end{abstract}

\section{EVOLUTION OF CLUMPY GAS IN GALAXIES}

\author{
R. Kunze, H.W. Yorke, R. Spurzem \\ Institut für Astronomie und Astrophysik, Universität Würzburg
}

The basic equations for treating a multicomponent medium: stars, gas clouds and an ambient gas are discussed elsewhere (Yorke, et al., this meeting). Here we apply the 2-D multicomponent code to the problem of explosions in a clumpy medium (cool "clouds" embedded in a warm $T \sim 10^{4} \mathrm{~K}$ or hot $T \sim 10^{6} \mathrm{~K}$ gas). The evolution of supernova remnants and supershells in media of varying degrees of clumpiness are compared and contrasted to the results of earlier calculations. 